ulcer cases. I deny that Dr. Carman sees his cases at the stage the rest of us do. Just recently, in reviewing my cases of ulcer, I was interested in noting how they ran as to time; and the stage at which they came to office consultation varied from six months to twenty years. I should like to have you go to the Mayo Clinic and find out whether Dr. Carman sees those cases in the first two or three or four months after the patient has complained of symptoms. His cases are not acute by any means.

\section{A CONTRIBUTION TO THE PROBLEM OF NEPHRITIS AND NEPHROSIS IN PREGNANCY *}

\section{JOSEPH L. BAER, M.D. CHICAGO}

Modern conceptions of renal disease from the standpoint of the physiologist, internist and pathologist are still far from clear. One need but read the publications of such keen observers as Volhard, ${ }^{1}$ Strauss, ${ }^{2}$ Christian, ${ }^{3}$ Mosenthal, ${ }^{4}$ Ringer, ${ }^{5}$ Richards, ${ }^{6}$ Kolischer, ${ }^{7}$ Morse ${ }^{8}$ and Epstein ${ }^{9}$ to realize this.

It seemed desirable, therefore, to select one angle as a working basis for study, and possibly for a revision of our views concerning the renal disturbances of pregnancy.

The kidneys excrete urine by means of two mechanisms, the vascular system, especially the glomeruli, which extract the water, and the tubular system, especially the granular cells in the capital sections of the tubules, which extract the urinary solids.

This division of function has led to a corresponding disease classification: nephritis, a disease of the vascular system, particularly the glomeruli, characterized by hematuria, macroscopic or microscopic, and a pronounced rise in blood pressure; and nephrosis, a pathologic involvement of the tubular system, characterized by albumin and casts, including epithelial casts, appearing in the urine, nitrogen and sodium chlorid retention and edema. Nephrosis of any considerable duration will always lead to pronounced cardiac changes with their associated symptoms. Mixed forms, that is, a combined nephritis and nephrosis, will occasionally be found in cases of long standing.

The nephropathy of pregnancy, the so-called "kidney of pregnancy," seems to occupy a position between nephritis and nephrosis. Very probably the initial changes occur in the renovascular system; and, because of the resulting dystrophy, later on the tubular epithelium becomes involved. The nephropathy of pregnancy is essentially a disease of primiparas. Any crowding of the ovum such as occurs in hydramnios or twins increases the disposition toward this disturbance. The association with increased intra-ovular pressure seems to indicate that the primary cause of this nephrop-

* Read before the Section on Obstetrics, Gynecology and Abdominal Surgery at the Seventy-Third Annual Session of the American Medical Surgery at the Seventy-Third An
Association, St. Louis, May, 1922 .

1. Volbard, F.: Die doppelseitigen haematogenen Nierenerkrankungen, Berlin, J. Springer, 1918.

2. Strauss, H.: Nephritiden, Nephrosen und Schrumpfniere, Berlin, Urban and Schwarzenber, 1920.

3. Christian, H. A.; Frothingham, C.,
Am. J. M. Sc. 150: 655 (Nov.) 1915.

4. Mosenthat, 5. Ringer, A. I.: Chronic Nephritis, Am. J. M. Sc. 161:798-818 (June) 1921 .

(Jan.) Richards. 1922 .

7.) Kolischer, G.: Urol. \& Cutan. Rev., 1921.

7. Kolischer, G.: Urol. \& Cutan. Rev.j 1921. 8. Morse, P. F.: The Correlation of Clinical and Patholog
ings in Nephritis, Am. J. M. Sc. 163:697-710 (May) 1922 .

9. Epstein, A. A.: The Therapeutic Management of Chronic Nephrosis, M. Clinics N. America 5: 1067.1079 (Jan.) 1922; (Clinical Types athy is a placental toxicosis. Its infrequency in subsequent pregnancies may be explained on the supposition that the system becomes immunized against this particular toxin. Prodromal symptoms, such as slight shifting edemas and moderate retention of sodium chlorid, are frequent. The condition occurs in the second half of pregnancy in the great majority of cases, and usually runs an insidious course. Most authors deny the transition of a pregnancy nephropathy into chronic nephrosis. Of course, the occurrence of pregnancy in a person with a preexisting nephrosis may, and as a rule will, aggravate the condition of renal decompensation.

In the study presented here, functional tests based on Mosenthal's ${ }^{10}$ pioneer work were employed, coupled with capillaroscopy. One of the renal functional tests, aimed at the tubular system, is made to discover what effect on the specific gravity of a series of two hourly specimens of urine follows the administration of a dyestuff such as methylene blue or indigocarmin.

Dyestuffs are excreted by way of the tubular system and can be seen passing through the epithelial cells. If these epithelial cells are diseased, the entrance of the dyestuff particles into the tubular system cripples the extractive power of the epithelial tubular lining.

In case of nephrosis, that is, an impairment of the tubules of the renal system, in which methylene blue has been employed, the extractive power will be affected in direct proportion to the extent of involvement of the tubular system; and since the tubular system supplies to the urine the chemicals which determine its specific gravity, successive readings of the specific gravity will tell us the tale of tubule involvement. The urine must always be boiled and filtered to remove the albumin.

In the normal case, administration of 5 grains of methylene blue and serial reading of the specific gravity of the urine thereafter shows a specific gravity unchanged or increased. In severe involvement, the specific gravity will drop suddenly and remain low for many hours.

Another test, "the freshet test," is aimed at the glomeruli. With a normal intake of liquid and an established quantity rate of excretion, the sudden drinking of a liter of water followed by quantity measurements of two hourly urinations reveals the condition of the renal vascular system. In the normal case, the extra liter will have been excreted in less than four hours. A nephritis will show delay; the more involvement, the more delay or "hyposthenuria." 11

The first extensive work in capillary microscopy was done by Lombard, ${ }^{12}$ an American, in 1912, followed by the work of two Germans, Mueller ${ }^{13}$ and Weiss, ${ }^{14}$ in 1916 , since which time clinicians, and laboratory workers generally, have taken up this method. The changes observed and most easily studied in the nail grooves fall into two groups: the transient, with slight deformities of the capillary loops, such as a bulge at the convexity, hairpin shapes and elongation, seen in the kidney of pregnancy and eclampsia $;^{15}$ and the perma-

10. Mosenthal, H. O.: Renal Function as Measured by the Elimination of Fluids, Saits and Nitrogen, and the Specific Gravity of the Urine, Arch. Int. Med. 16:733, 1915. 11. Koranyi, F.: Diagnostische Irrtuemer in Erkrankungen der 12. Lombard: The Blood Pressure in the Arterioles, Capillaries Small Veins of the Human Skin, Am. J. Physiol. 24:355, 1912. 13. Mueller and Weiss: Ueber Beobachtung der Hautkapillaren th ihne klinische Bedeutung, Munchen. med. Wchnschr. 64: 609, 1917 . der Hautkapillaren am lebenden Menschen, Deutsch. Arch. f. klin. Med. 119: $1-38,1916$.

15. Hinselmann: Untersuchungen über die Eklampsia, Zentralbl f Gynäk. 44:987 (Sept. 4) 1920. Nevermann: Zentralbl. f. Gynäk. 44: 
nent, with meandering loops, tennis racket forms and retardation of the blood stream, with segmentation, seen in pronounced true nephrosis, especially if combined with general circulatory disturbances due to the renal changes, and also in sclerosis of the kidney, more particularly the rapidly contracted kidney.

The differential diagnosis between nephropathy independent of pregnancy and nephropathy due to pregnancy is based on the interpretation of renal and remote symptoms plus capillaroscopy.

There is no hyposthenuria in the nephropathy of pregnancy. The kidneys have not lost their elasticity of function; they are still able to adjust themselves immediately to a sudden increased demand due to excessive intake of fluid. The passage of stains through the kidney by administration of methylene blue or indigocarmin will not cause a sudden drop in the specific gravity of the urine as is the case in genuine nephrosis. The capillary changes are of the transient type. There are no changes in the retina, and the pronounced cardiac changes so common in genuine chronic nephrosis are missing. Further differential points are the time of onset of the renal disturbance in the course of the pregnancy and the parity of the patient.

The treatment of the kidney of pregnancy should be determined by our improved knowledge of renal physiology and pathology and the relative predominance of nephritic or nephrotic symptoms. While we are not in a position to influence renal diseases by direct and specific medication, we can hasten recovery by relieving the diseased structures. If, for example, the involvement of the glomeruli predominates, the intake of fluid must be reduced to a minimum during the acute stage. The glomeruli attend mainly to the extraction of water, and any additional fluid intake would mean more work for a structure already weakened. If, however, the epithelial involvement is to the fore, a decided increase of fluids and the administration of diuretics will prevent retention of solids, because the increased amount of urine will make up for the reduction of the concentrating power of the affected kidneys.

The nephropathy of pregnancy does not furnish an indication for artificial interruption of pregnancy, but a genuine nephrosis of marked degree under certain conditions necessitates emptying the uterus. Among such indications may be mentioned retinitis albuminuria, or edema of such a degree or location as to be vital.

Idiopathic pyelitis, that is, infection and inflammation confined to the renal pelvis, occurs frequently in pregnancy. The highest figures indicate that 50 per cent. of all pregnant women are affected. It is a "Stauung" disease; that is, stagnation and damming back, the invading germ as a rule being the colon bacillus. It may become very severe, and occasionally pus and blood appear in such quantities as to give rise to the suspicion of an involvement of the renal parenchyma itself, a true pyonephrosis. The differential diagnosis is made by testing the integrity of the renal function. True pyelitis is clinically cured by a restoration of free drainage, either by the insertion of the ureteral catheter, or, in appropriate cases, by postural treatment of the patients. This is based on the observation that the obstruction of the ureter by compression invariably occurs above the pelvic brim where the ureter crosses the quadratus lumborum. Placing the patient on the side of the affected ureter causes the uterus to dislodge the fetal pole which is responsible for the compression.
Eclampsia and uremia warrant some special discussion. While there is a certain similarity as to etiology and symptoms, they are quite distinct entities. Both are based on the presence of certain toxins in the circulation, but these toxins are of a widely different character. ${ }^{16}$ This is proved by the temperature reaction. Eclampsia is always accompanied by a rise in temperature; the opposite is true of uremia. The depressing effect of the uremic poison on the thermic centers is so decided that, when a virulent pus infection occurs during a uremic process, there is usually no fever, though under ordinary circumstances such an infection would cause alarming rises in temperature. That eclampsia is not dependent on nephritis or nephrosis, and that the toxicosis is different from that of uremia is further evidenced by the fact that, in the fatal eclampsias without any convulsions, the postmortem reveals the changes characteristic of eclampsia, which are never seen in necropsies in cases of kidney disease. Biologists and pathologists give as the cause of uremia the presence of so-called extractive materials, soluble in alcohol and ether, chemically not well defined but evidently retained protein split products; while as the cause of eclampsia, they speak of placental toxins, very probably biogenous amins. The occurrence of eclampsia with hydatid mole is further evidence that the chorionic villi are the actual source of the toxin.

Both uremia and eclampsia present the symptoms of increased intracranial and intraspinal pressure. Whether the convulsions, amaurosis and coma are due to edema of the brain or to angiospasm is still open to discussion. Th latter theory is supported by the observation that attacks are frequently cut short by therapeutic interference. The quickness with which improvement sometimes occurs certainly does not allow time for the draining off of a cerebral edema, but allows ample time for the release of an angiospasm.

With the diagnosis of eclampsia established, the principles of treatment are clear: (1) to detoxify, and thus prevent convulsions and further injury to kidneys and liver, and (2) to remove the source of the toxin, the placenta. Williams ${ }^{17}$ and Balard ${ }^{18}$ have found a single vigorous bloodletting a sufficient measure to satisfy the requirements in most cases. The Swiss ${ }^{13}$ clinics are again advocating cesarean section. The Michael Reese clinic has for some years used the VeitStroganoff cycle of morphin and hydrated chloral (latterly substituting phenobarbital for chloral) plus purging, sweating, and venesection for high pressure, to accomplish the detoxifying, and conservative measures to favor emptying of the uterus. The action of the sedatives in suppressing the convulsions may be explained on the assumption that they relax the cerebral angiospasm.

Prognosis in nephrosis is better than in nephritis, since the epithelium of the tubular system has a regenerative power which is lacking in the glomerular system. Recovery of renal function in the kidney of pregnancy and in eclampsia is usually complete.

REPORT OF ILLUSTRATIVE CASES

CASE 1.-Kidney of pregnancy, mild. Mrs. C., a primipara, aged 28, developed albuminuria in the sixth month, with no casts and a blood pressure of 148, systolic, and 104 , diastolic. At term, examination of the urine revealed: albumin, $1+$; casts negative; some epithelial cells and white

\footnotetext{
16. Alder: Schweiz. med. Wchnschr. 51:713 (Aug. 4) 1921.

17. Williams, J. W.: Personal communication to the author.

16: 257 (May)

19. Huessey, P.: Schweiz. med. Wchnschr. 51: 845, 874, 899. 1921
} 
blood cells; specific gravity, 1.020 ; methylene blue readings: 1.020-1.018-1.022-1.020; blood pressure, 160, systolic, 94. diastolic; no hyposthenuria. Capillaroscopy revealed that the loop arms were even. There was some elongation, and the flow was even. The changes were of the transient type. At labor, the albumin was $1+$; the casts were negative; the blood pressure was: 190 , systolic, 110 diastolic. The blood pressure taken after delivery (by low forceps) was 166, systolic, 94 diastolic.

This was a mild toxemia promptly relieved by delivery.

CASE 2. - Nephrosis in pregnancy. Mrs. B., a tertiodecipara, aged 42, suffered from double mitral and aortic regurgitation. At the eighth month of pregnancy, examination of the urine revealed: specific gravity, 1.018; albumin, $2+$; casts, granular and epithelial; blood pressure: 172 systolic, 110 diastolic. There was some dyspnea and decompensation. At term, the specific gravity was 1.024 , and the albumin, $3+$. Casts were numerous, granular and epithelial. Methylene blue readings were: 1.024-1.024-1.018-1.021-1.021. Hyposthenuria was present more than twelve hours. Capillaroscopy revealed that all loops were tortuous, meandering, elongated, and tennis racket shape. The flow was visible and there was no retardation. The changes were of the pcrmanent type. Delivery was spontaneous, in two hours.

This was a chronic nephrosis with circulatory changes. The tubular system was still equal to its task.

CASE 3. - Kidney of pregnancy, severe. Mrs. L., primipara. aged 29 , in the last months of pregnancy had albumin, $1+$ in the urine, many leukocytes and epithelial cells. but no casts, a specific gravity of 1.018 and a blood pressure of 148 systolic, 90 diastolic. Methylene blue readings were: 1.012-1.008-1.006-1.010-1.012. Capillaroscopy revealed slightly tortuous loops, many hairpin forms and some elongation. Changes were of the transient type. The patient was vigorously purged and sweated, and the next series showed normal urine, with a specific gravity of 1.030. Methylene hlte readings were: 1.011-1.014-1.011-1.013-1.018. Capillary lindings remained unchanged. The blood pressure was 140 systolic, 90 diastolic. Four days iater, the patient went into spontaneous labor, and in two hours and ten minutes was delivered uneventfully of a small, healthy baby. On the tenth day after delivery, the specific gravity was 1.010 , and after methylene blue had been given, 1.008-1.006-1.009-1.006.

In this patient, it seemed that the tubule function was on the verge of a breakdown, as indicated by the fall in specific sravity when the dyestuff particles crippled the epithelial celis.

CAse 4.-Previous eclampsia, vulnerable tubule system. Mrs. W., secundipara, aged 31, had eclampsia at the end of her lirst pregnancy (1919). The convulsions, two in number, octurring four days before delivery, were controlled by the administration of morphin and chloral. Vigorous measures for elimination were instituted; the patient went into spontaneous labor, and was delivered by low forceps. One month before the second delivery, examination of the urine revealed: specific gravity, 1.012; no albumin and no casts. Methylene blue readings were: 1.002-1.006-1.008. At term, the albumin was $1+$; there were a few hyaline casts, and the specific gravity was 1.021. After administration of methylene blue, the specific gravity was: 1.009-1,005-1.002-1.009-1.013; blood pressure, 120 systolic, 78 diastolic. There was no hyposthenuria. Capillaroscopy revealed: vessels slightly tortuous and elongated; visible flow, somewhat uneven; the transient type. Delivery was spontaneous.

This patient apparently had a vulnerable tubule system, as indicated by the lowering of the specific gravity; yet she did not develop eclampsia again, but had a spontaneous delivery.

CASE 5. - Kidney of pregnancy, nephrotic type. Mrs. T., tertipara, aged 27 , whose previous labors were normal, in late pregnancy had an albuminuria $(3+)$. The urine was dark straw colored; specific gravity, 1.021; and there were numerous granular casts. Methylene blue readings were 1.023-1.022-1.022-1.028. Blood pressure was 162 systolic, 80 diastolic. The patient was purged and sweated. Three days liter, the urine showed $4+$ albumin; innumerable hyaline and granular casts, specific gravity 1.028; blood pressure, 140 systolic, 110 diastolic. Capillaroscopy revealed only slight changes; elongation and hairpin forms with no stasis; a transient type. Labor was induced by bag insertion.

That this was a kidney of pregnancy and not a nephrosis was evidenced by the absence of albuminuric retinitis, edema and cardiac changes.

\section{SUMMARY}

1. Nephritis and nephrosis are separate and welldefined renal diseases involving different parts of the kidney mechanism, with different findings, and requiring different treatment.

2. Renal function tests and capillaroscopy are fully as important as urinalyses and blood pressure readings and are necessary to classify and treat renal pathologic conditions in pregnancy.

3. The kidney of pregnancy occupies a position between nephritis and nephrosis, can be differentiated and should be treated like the type it resembles.

4. Pyelitis of pregnancy of severe degree can be differentiated from a pyonephrosis by testing the integrity of the renal function.

5. Eclampsia seems to be due to a placental toxin which causes a special toxicosis of the brain and consequent convulsions, totally different from the uremia of nephrosis, from which it can best be distinguished by the accompanying fever, which is absent in uremia.

104 South Michigan Avenue.

\section{ABSTRACT OF DISCUSSION}

Dr. A. B. Spalding, San Francisco: Dr. Addis developed a method of quantitative kidney function tests which was based on the observation that the function of the kidney is dependent on and becomes a measure of the amount of renal tissue it contains. When the kidney is placed under strain by a patient's having been given $20 \mathrm{gm}$. of urea and water, the kidney is placed at its maximal capacity, and if the urine is drawn off each hour and 100 c.c. of blood is withdrawn in the interval, the ratio of urine urea to blood urea can be obtained. Giving more and more urea to a patient shows that the amount of urea found in the urine increases exactly proportionate to that amount. We applied this test to 163 normal men and found that the average ratio was 50.4 per cent., which has been adopted as a standard for normal renal tissue. We have applied this test to various conditions in pregnancy. In eleven normal patients in the latter months of pregnancy, there was an increase in the ratio and, therefore, it is justifiable to assume that there is hypertrophy of the kidney during normal pregnancy. In testing certain cases of pregnancy nephrosis, it was interesting to find that in all but one case there was only a moderate reduction in kidney function. With patients even with marked toxic symptoms, so far as the kidney is concerned, there is no danger of serious renal dysfunction. If we are going to treat the patients, it is the toxemia that must be treated, and not the kidney lesion. The kidney has six times more normal tissue than is needed. In applying this test to patients after they have suffered from a pregnancy toxemia, excluding cases of chronic nephritis, we were surprised to find in the majority of cases that there was a progressive loss of kidney function. That is the important thing. We have observed one patient four years. This patient did show some vascular changes, but otherwise the case looked like a fatal nephrosis. We believe that the patient received her kidney lesion in her first pregnancy, and lost her life from the damage to the tubules that persisted after pregnancy. The point I wish to bring out in this discussion is that we must have quantitative methods for estimating kidney function. Qualitative methods are good, but not sufficient.

Dr. Cart H. Davis, Milwaukee: The one point that has been most overlooked by men in general practice particularly is the importance of making a microscopic examination of 
the urine whenever there is even a slight trace of albumin. This is especially true during pregnancy. If one examines a centrifuge specimen, the probabilities are that one will find a considerable number of pus cells, so that the important test in many cases is a low power microscopic examination of the uncentrifuged specimen. If in such an examination one finds a considerable number of pus cells, securing a catheterized specimen or otherwise, one should rule out the possibility of vaginal contamination. A centrifuged specimen is necessary in looking for casts. I was called to see a patient in the seventh month of pregnancy. A commercial laboratory was making all sorts of tests at considerable expense to the patient. It had constantly reported the presence of albu$\min$ in the urine. But the commercial laboratory did not use a little common sense and make a microscopic examination because that had not been called for. Owing to the type of temperature, etc., a diagnosis of typhoid had been made by the physician in charge. He had placed the patient on the starvation diet, and she was failing rapidly. A carefully taken history revealed that she had given symptoms suggesting an acute infection of the kidney, and the uncentrifuged drop of urine under the microscope showed that the urine was loaded with pus cells. Many cases are overlooked because a simple test has not been made. We should be more careful in checking up, whenever there is a trace of albumin in these cases, ruling out the possibility of vaginal contamination and determining what the condition of the urine really is.

Dr. N. S. Heany, Chicago: I should like to have Dr. Baer tell us whether he can correlate the findings by his methods with the findings obtained by Dr. Spalding, and whether they run parallel. There is a suggestion that this is not the case Are these two methods workable together? I should like to have him tell us also about capillaroscopy.

Dr. JoSEPH L. BAER, Chicago: Concerning Dr. Spalding's excellent renal function test: I had considerable difficulty in selecting a test that would be simplest to handle and most satisfactory in result. The two I selected-the dye-stuff test, aimed at the tubules, and the freshet test, aimed at the glomeruli-coupled those two requirements, and to a certain degree both are quantitative proportionally to lowering of specific gravity and retention of the liter of water. Dr. Spalding spoke about the ultimate death four years after confinement of a patient with toxemia of pregnancy with renal involvement. The prognosis in nephrosis is decidedly better than in nephritis, because the tubules have a regenerative power which is lacking in the glomeruli. If the toxic kidney leans toward the tubular type, its recovery can be complete; if toward the glomerular type, it is going to be rather badly damaged. Dr. Davis' comment on the necessity for studying the microscopic sediment requires no further comment on my part. Answering Dr. Heaney's inquiry as to the capillaroscopy, the best place for study is at the nail groove, finger or toe. The index finger lightly strapped to a wooden tongue depressor is secured on the stage of the microscope. A small bulb at one side of the finger nail gives reflected light. The skin must be made translucent by emersion oil. The normal capillaries around the nail groove show a soldier-like uniformity. They run in parallels. The loop ends are rounded like the bases of well-formed test tubes. The transient type of involvement first produces hair-pin shapes, some elongation of the capillaries, long ones next to short ones, and a bulge at the convexity. The caliber at the loop is increased. As the condition becomes permanent, meandering loops are seen. They assume grotesque shapes, such as figure eights. Some observers describe anastomosis, also tennis racquet shapes at the loop. The normal capillary stream is perfectly visible in these loops. Some assert that it has a definite rhythm in common with the respiration. With a pause in the respiration there is a pause in the capillary circuit. With inspiration, the capillary circuit moves on again. In advanced chronic nephrotics with circulatory disturbance, in sclerosis of the kidney and other deep-seared changes, there is a retardation in the blood stream. It can be seen stagnant in the capillary loops and segmented like the broken mercury column in a thermometer. Retardation and segmentation are striking changes. Lastly, in eclampsia there is apparently a complete obliteration of the lumen of the arterial arm and a wide dilatation of the venous arm.

\section{QUARTZ LIGHT THERAPY IN SKIN DISEASES *}

\section{E. LAWRENCE OLIVER, M.D. BOSTON}

The term quartz light as generally used refers to a mercury vapor light in a fused quartz vacuum tube, the mercury making the arc. Such a lamp is very rich in ultraviolet light; and, as quartz to a large degree is transparent to ultraviolet rays, these rays emerge from the lamp and can be utilized for therapeutic purposes.

As to the nature of ultraviolet light: When sunlight is passed through a prism, it is split up into many rays. We are all familiar with the visible rays of color so produced, but at each end of the visible spectrum there are innumerable invisible rays. At one end of the visible spectrum we have the invisible infra-red of longer and longer wave lengths the farther we go beyond the visible red, while at the other end beyond the visible violet we have the invisible ultraviolet, of shorter and shorter wave length the farther we go beyond the violet.

In physics, light and electricity were formerly taught as separate subjects; but today light is regarded as an electromagnetic phenomenon, and may therefore be considered as a form of electricity. The electromagnetic waves of different lengths have different properties. The shortest wave lengths known are those of the gamma rays of radium, which are measured in quintillionths of an inch, while the waves utilized in wireless and radio transmission reach many miles in length.

The ultraviolet rays which we are considering are sometimes called chemical rays, as they seem to have a chemical action. On the skin they produce what is commonly called sunburn. Now, naturally we would suppose that sunburn is caused by the hot waves of light; but the contrary is the truth. It is the cold, invisible ultraviolet light that produces sunburn. This is easily proved by laying a piece of ordinary glass on a portion of the skin exposed to sunlight and observing that the skin beneath the glass does not become sunburned. The glass is transparent to all the visible and heat waves of sunlight, but it blocks the ultraviolet rays. If, on the other hand, we substitute quartz glass, which is transparent to ultraviolet light, sunburn will occur.

The quartz lamps in use at present are of two types, air cooled and water cooled. With the air cooled lamps, large areas of skin may be treated with a single exposure; but, on account of the heat of these lamps, they must be used at a distance of 6 inches or more. For intensive effect on small areas, the water cooled lamps are more effective. In the water cooled lamps the quartz tube is surrounded with a water jacket of running water keeping the lamp constantly cool, so that the window of the lamp may be pressed directly against the skin, bringing the source of light only about an inch away from the skin. Not only does this have the advantage of close approximation of the light, but also the pressure of the quartz glass dehematizes the skin so that the rays have a deeper penetration.

* Read before the Section on Pharmacology and Therapeutics at the Seventy-Third Annual Session of the American Medical Association, St. Louis, May, 1922. 